\title{
LncRNA-HGBC stabilized by HuR promotes gallbladder cancer progression by regulating miR-502-3p/SET/AKT axis
}

Yun-ping $\mathrm{Hu}^{1,2,3,4 \dagger}$, Yun-peng Jin ${ }^{1,2,3 \dagger}$, Xiang-song $\mathrm{Wu}^{1,2,3 \dagger}$, Yang Yang ${ }^{1,2,3}$, Yong-sheng $\mathrm{Li}^{1,2,3}$, Huai-feng Li ${ }^{1,2,3}$, Shan-shan Xiang ${ }^{1,2,3}$, Xiao-ling Song ${ }^{1,2,3}$, Lin Jiang ${ }^{1,2,3}$, Yi-jian Zhang ${ }^{1,2,3}$, Wen Huang ${ }^{1,2,3}$, Shi-li Chen 1,2,3, Fa-tao Liu ${ }^{1,2,3}$, Chen Chen ${ }^{1,2,3}$, Qin Zhu ${ }^{1,2,3}$, Hong-zhuan Chen ${ }^{4^{*}}$, Rong Shao ${ }^{1,4^{*}}$ and Ying-bin Liu ${ }^{1,2,3^{*}}$ (D)

\begin{abstract}
Backgrounds: Long non-coding RNAs (IncRNAs) are essential factors that regulate tumor development and metastasis via diverse molecular mechanisms in a broad type of cancers. However, the pathological roles of IncRNAs in gallbladder carcinoma (GBC) remain largely unknown. Here we discovered a novel IncRNA termed IncRNA Highly expressed in GBC (IncRNA-HGBC) which was upregulated in GBC tissue and aimed to investigate its role and regulatory mechanism in the development and progression of GBC.

Methods: The expression level of InCRNA-HGBC in GBC tissue and different cell lines was determined by quantitative real-time PCR. The full length of InCRNA-HGBC was obtained by $5^{\prime}$ and $3^{\prime}$ rapid amplification of the cDNA ends (RACE). Cellular localization of IncRNA-HGBC was detected by fluorescence in situ hybridization (FISH) assays and subcellular fractionation assay. In vitro and in vivo assays were preformed to explore the biological effects of IncRNA-HGBC in GBC cells. RNA pull-down assay, mass spectrometry, and RNA immunoprecipitation (RIP) assay were used to identify IncRNA-HGBC-interacting proteins. Dual luciferase reporter assays, AGO2-RIP, and MS2RIP assays were performed to verify the interaction between IncRNA-HGBC and miR-502-3p.

Results: We found that IncRNA-HGBC was upregulated in GBC and its upregulation could predict poor survival. Overexpression or knockdown of InCRNA-HGBC in GBC cell lines resulted in increased or decreased, respectively, cell proliferation and invasion in vitro and in xenografted tumors. LnCRNA-HGBC specifically bound to RNA binding protein Hu Antigen R (HuR) that in turn stabilized IncRNA-HGBC. LncRNA-HGBC functioned as a competitive endogenous RNA to bind to miR-502-3p that inhibits target gene SET. Overexpression, knockdown or mutation of IncRNA-HGBC altered the inhibitory effects of miR-502-3p on SET expression and downstream activation of AKT. Clinically, IncRNA-HGBC expression was negatively correlated with miR-502-3p, but positively correlated with SET and HuR in GBC tissue.
\end{abstract}

Conclusions: Our study demonstrates that IncRNA-HGBC promotes GBC metastasis via activation of the miR-5023p-SET-AKT cascade, pointing to InCRNA-HGBC as a new prognostic predictor and a therapeutic target.

Keywords: Gallbladder cancer, IncRNA-HGBC, miR-502-3p, SET, HuR

\footnotetext{
*Correspondence: yaoli@shsmu.edu.cn; rongshao@sjtu.edu.cn; liuyingbin@xinhuamed.com.cn

†Yun-ping Hu, Yun-peng Jin and Xiang-song Wu contributed equally to this work.

${ }^{4}$ Department of Pharmacology, Shanghai Jiao Tong University School of Medicine, W. Building 3, Room 407, 280 Chongqi Road, Shanghai 200025, China

${ }^{1}$ Department of General Surgery, Xinhua Hospital, Affiliated to Shanghai Jiao Tong University School of Medicine, Building 25, Room 513, 1665 Kongjiang Road, Shanghai 200092, China

Full list of author information is available at the end of the article
} 


\section{Introduction}

Gallbladder cancer (GBC) is a rare, but is an extremely aggressive carcinoma developed from the biliary tract [1]. Regardless of the relatively higher incidence in Asia than in Western countries [2], almost GBC cases are diagnosed at advanced stages due to the lack of early symptoms. Thus, most of those patients fall into unexpected contraindication of the resection, while the rest need immediately surgical removal [3-5]. To date, adjunctive therapy including chemo- and radiotherapy is an indispensable regimen to treat the majority of GBC patients. However, the efficacy of those conventional therapies is transient and barely minimal, as most of the patients rapidly recur and concomitantly develop chemo- and radio-resistance [6]. Hence, the overall prognosis of $\mathrm{GBC}$ is extraordinarily poor and the mean survival ranges from 13.2 to 19 months [7, 8]. Although over the last decades large effort has been made in identification of tumor-promoting oncogenes and tumor suppressors in GBC, there is still lack of independent biomarkers that can be routinely used in clinical practice $[9,10]$. Therefore, it is of paramount importance to identify novel factors that potentially serve as novel diagnostic biomarkers and therapeutic targets for the treatment of patients with GBC.

Long non-coding RNAs (lncRNAs) comprise a wide variety of ncRNA species with a minimum length of 200 nucleotides(nt) but deficiency of protein-coding potential [11-13]. LncRNAs share many characteristics with mRNAs in which they are transcribed by RNA polymerase II and can be capped, spliced, and polyadenylated. LncRNAs are located in intergenic DNA, introns, or overlapping with other genes in an antisense orientation, and their expression is usually restricted to specific tissue. Emerging lines of evidence have shown that lncRNAs are involved in diverse biological processes and are key regulators in pathologic process at both transcriptional and post-transcriptional level [14-16]. It is noted that, a new regulatory mechanism termed competing endogenous RNAs (ceRNAs) has been identified to mediate lncRNA activity. In this model, lncRNA or mRNA harboring the same miRNA response element (MRE) can modulate each other's expression levels by competitively binding to shared miRNAs that block target mRNAs [17]. In cancer, it is appreciated that lncRNAs can contribute to tumor progression and drug resistance [18-20]. For example, the lncRNA PCA3 has been identified as a novel diagnostic marker of prostate cancer progression [21] and simultaneously its lncRNAbased therapies are also formulated [22]. However, it largely remains unknown with regards to a variety of novel lncRNAs in the pathological regulation of GBC.

In the current study, based on our previous microarray data analysis, we identified a new lncRNA highly expressed in GBC, termed lncRNA highly expressed in gallbladder cancer (lncRNA-HGBC) as a key regulator of GBC growth and metastasis. We demonstrate that lncRNA-HGBC specifically binds to RNA binding protein HuR that in turn stabilizes IncRNA-HGBC. In addition, lncRNA-HGBC regulates miR-502-3p/SET/ $\mathrm{AKT}$ axis by directly binding to and sequestering miR502-3p that inhibits SET gene expression, thereby leading to the activation of AKT downstream pathway. To this end, our findings establishing HuR/lncRNA-HGBC/ miR-502-3p/SET/AKT regulatory axis may offer novel targets for $\mathrm{GBC}$ therapy.

\section{Material and methods}

\section{Patients and clinical specimens}

Human GBC samples and adjacent benign gallbladder tissues were obtained from patients who underwent cholecystectomy without receiving preoperative chemotherapy, radiotherapy, or androgen therapy at the Department of General Surgery, Xinhua Hospital, School of Medicine, Shanghai Jiao Tong University between 2008 and 2013. Written informed consent was obtained from all participants. This study was approved by the ethics committee of Xinhua hospital. For quantitative real-time PCR analysis (qRT-PCR), tumor tissues as well as the adjacent non-tumor tissues were snap-frozen in liquid nitrogen and stored at $-80^{\circ} \mathrm{C}$. For immunohistochemical staining, each tissue sample was fixed in $4 \%$ formalin immediately after removal and embedded in paraffin.

$5^{\prime}$ and $3^{\prime}$ rapid amplification of CDNA ends (RACE) analysis Total RNA was isolated using TRIzol Plus RNA Purification Kit (Invitrogen), according to the manufacturer's instructions. 5' RACE and 3' RACE were performed using GeneRacer $^{\mathrm{TM}}$ Kit (Invitrogen) according to the manufacturer's instructions. The following gene-specific primers (GSP) are used for PCR: 5'-CCCCTGGAGGAGGT GGAGCTTACAGAA-3' (5' RACE GSP1), 5'-GTGGCT CATGCCTGTAATCCCAACACTTT-3' (5' RACE GSP2), 5' -CCAGGTTAGTTCCTTCTGTAAGCTCC ACCTC-3' (3' RACE GSP1), 5'-CACCCGCTAATTGG CTCCCTCAGATC-3' (3' RACE GSP2).

\section{Northern blot analysis}

Northern blot was performed to characterize the full length of lncRNA-HGBC as previously described [23] with minor modifications. Briefly, $30 \mu \mathrm{g}$ of indicated RNA was separated by formaldehyde gel electrophoresis and then transferred to a Biodyne Nylon membrane (Pall, NY, USA) and fixed by UV crosslinking. After prehybridization in Ultrahyb buffer (Ambion, Grand Island, $\mathrm{NY}$ ) at $62{ }^{\circ} \mathrm{C}$ for $60 \mathrm{~min}$, the membrane was hybridized in Ultrahyb buffer with digoxin-labeled probes for lncRNA-HGBC or $\beta$-actin at $62^{\circ} \mathrm{C}$ overnight. The 
membrane was then washed 2 times with $2 \times$ SSC at $62{ }^{\circ} \mathrm{C}$ for $5 \mathrm{~min}$ and incubated with anti-DIG-biotin antibody (BOSTER Biological Technology, BM0040) for $2 \mathrm{~h}$ at RT. Then the membrane was washed 2 times with $2 \times \mathrm{SSC}$ at $62^{\circ} \mathrm{C}$ for $5 \mathrm{~min}$ and incubated with HRPconjugated Streptavidin (BOSTER Biological Technology, BA1088) for $30 \mathrm{~min}$ at RT. After washing for another 2 times with $2 \times$ SSC, the expression of lncRNAHGBC was detected. The probe sequences were listed in Additional file 1: Table S1.

\section{Fluorescence in situ hybridization}

Fluorescence in situ hybridization (FISH) was performed as previously described [23]. Briefly, NOZ and SGC-996 cells grown on the slides were washed with PBS and fixed in $4 \%$ paraformaldehyde. After protease reagent treatment, the slides were incubated with prehybridization buffer at $40^{\circ} \mathrm{C}$ for $4 \mathrm{~h}$, and then hybridized with digoxin-labeled probe at $40^{\circ} \mathrm{C}$ overnight. After washing and blocking, the slides were incubated with biotin conjugated anti-digoxin antibody. The slides were then incubated with SABC-FITC at $37^{\circ} \mathrm{C}$ for $30 \mathrm{~min}$ after washing. The images were captured using a confocal microscope. The probe sequence was listed in Additional file 1: Table S1.

\section{In vitro translation}

Transcription and translation assays were performed using TNT $^{\oplus}$ T7 Quick Coupled Transcription/Translation Systems and Transcend ${ }^{\mathrm{TM}}$ Non-Radioactive Translation Detection Systems (Promega) according to the manufacturer's instructions. Briefly, $1 \mu \mathrm{g}$ pBluescript II SK-HGBC plasmids or $1 \mu \mathrm{g}$ T7 Luciferase Control DNA(positive control) were assembled appropriately in a $0.5 \mathrm{ml}$ micro-centrifuge tube in the presence of BiotinLysyl-tRNA. Then the mixture was incubated at $30^{\circ} \mathrm{C}$ for $90 \mathrm{~min}$. The translation products were subjected to SDS-PAGE using 4-20\% gradient gel and transferred to PVDF membrane. After blocking, the membrane was incubated with an anti-Streptavidin-HRP antibody at $4{ }^{\circ} \mathrm{C}$ overnight. The signal was detected using ECL reagent.

\section{Dual-luciferase reporter assay}

Based on bioinformatic prediction [24], 6 miRNAs were selected as candidate targets of lncRNA-HGBC. pmirGLO Dual-Luciferase miR Target Expression Vector (Promega) was used to assess the direct binding of potential miRNAs to IncRNA-HGBC. The wild-type reporter construct pmirGLO-HGBC or the mutant reporter construct pmirGLO-HGBC-mut(miR-502-3p) was cotransfected with miR-502-3p mimic or miR-Control in $293 \mathrm{~T}$ cells. After transfection for $24 \mathrm{~h}$, Firefly luciferase levels were measured using a Dual-Luciferase Reporter Assay System (Promega,
Wisconsin) and normalized to Renilla luciferase activity. Each experiment was repeated at least three times.

\section{RNA pull-down and mass spectrometry}

Biotin-labelled lncRNA-HGBC was first transcribed in vitro from pBluescript II SK-lncRNA-HGBC using Biotin RNA Labeling Mix (Roche, Germany) by SP6(for antisense)/T7(for sense) RNA polymerase (Roche) according to the manufacturer's instructions. The RNA products were treated with RNase-free DNase I (Roche) and purified with an RNeasy Mini Kit (Qiagen, Valencia, CA). Four microgram biotinylated RNAs were denatured for $5 \mathrm{~min}$ at $65^{\circ} \mathrm{C}$ in PA buffer $(10 \mathrm{mM}$ Tris $\mathrm{HCl} \mathrm{pH} 7.5,10 \mathrm{mM}$ $\mathrm{MgCl} 2,100 \mathrm{mM} \mathrm{NH} 4 \mathrm{Cl}$ ) and slowly cooled down to room temperature. Then, the folded RNA was incubated with streptavidin Dynabeads (Invitrogen) for $1 \mathrm{~h}$ at $4{ }^{\circ} \mathrm{C}$ in the presence of $2 \mathrm{U} / \mathrm{ml}$ RNasin (Promega). After washing $4 \times$ 5 min with wash buffer (10 mM HEPES pH 7.0, $400 \mathrm{mM}$ $\mathrm{NaCl}, 1 \mathrm{mM}$ DTT, 1\% Triton X-100, protease inhibitor cocktail (Roche), $2 \mathrm{mM}$ RVC), the protein lysate from $1 \times$ $10^{7} \mathrm{NOZ}$ cells was pre-cleared by streptavidin Dynabeads (Invitrogen) and incubated with the folded RNA-beads complex for $3.5 \mathrm{~h}$ at $4{ }^{\circ} \mathrm{C}$ in the presence of $20 \mu \mathrm{g} / \mathrm{ml}$ yeast tRNA. After extensive washing, beads were boiled $40 \mu \mathrm{l}$ of $1 \times$ SDS loading buffer for $10 \mathrm{~min}$ at $100{ }^{\circ} \mathrm{C}$. The lncRNAinteracting proteins were further separated by sodium dodecyl sulphate-polyacrylamide gel electrophoresis and the gel was silver stained. Then, lncRNA-HGBC specific bands were subjected to mass spectrometry and retrieved in human proteomic library.

\section{Isolation of cytoplasmic and nuclear RNA}

Cytoplasmic and nuclear RNAs of NOZ cells were extracted and purified using PARIS ${ }^{\mathrm{TM}}$ Kit (Invitrogen) according to the manufacturer's instructions.

\section{RNA immunoprecipitation (RIP) assay}

RIP was performed using a Magna RIP RNA-Binding Protein Immunoprecipitation kit (Millipore, Bedford, MA) according to the manufacturer's instructions. Briefly, $2 \times 10^{7} \mathrm{NOZ}$ cell lysates were incubated with magnetic beads conjugated with negative control normal mouse IgG or human anti-Ago2 antibody (Millipore). The immunoprecipitated RNAs were then extracted and detected by qRT-PCR to confirm the enrichment of binding targets and the products were then subjected to agrose gel electrophoresis. The primers used for detecting lncRNA-HGBC or miR-502-3p were listed in Additional file 1: Table S2.

\section{MS2-RIP}

We co-transfected pcDNA3.1-MS2, pcDNA3.1-MS2HGBC, pcDNA3.1-MS2-HGBC-MUT(502-3p) along with pMS2-GFP(Addgene) into NOZ cells using Viafect reagent. 
After $48 \mathrm{~h}$, cells were collected and lysed to perform RNA immunoprecipitation (RIP) experiments using a GFP antibody (Roche) and the Magna RIP ${ }^{\mathrm{T} M}$ RNA-Binding Protein Immunoprecipitation Kit (Millipore, Bedford, MA) according to the manufacturer's instructions. Finally, purified RNAs were isolated and determined by real-time PCR to confirm the presence of binding targets. The primers used for detecting miR-502-3p or miR-122 were provided in Additional file 1: Table S2.

\section{Statistical analysis}

All statistical analyses were performed using SPSS 19.0 software. Each experiment was performed in triplicate, and the data were shown as the mean \pm SD, unless otherwise stated. Kaplan-Meier analysis with log-rank test was used for survival analysis. Student's t-test was used to compare the mean values. Pearson chi-square test was used to analyze the association between lncRNA-HGBC expression and clinicopathologic parameters. $P$ value $<0.05$ was considered to be statistically significant.

\section{Supplemental materials and methods}

Supplemental Materials and Methods were provided as Additional file 2 and Additional file 1: Table S1-S3.

\section{Results}

LncRNA-HGBC was identified and its high expression was correlated with poor prognosis of GBC

We previously performed a microarray analysis to compare both lncRNA and mRNA differential expression profile between GBC and adjacent benign tissues [23] (Gene Expression Omnibus(GEO) accession number: GSE76633). In the top candidate lists of differentially expressed lncRNAs, we noted that a lncRNA labeled as NR_027005, which has a high rate of coexpressed protein-coding RNAs, was increased by 16.6 times in GBC tissue relative to non-tumor tissue (Additional file 3: Figure S1A). Therefore, we were particularly focused on this uncharacterized lncRNA and named lncRNA-HGBC (lncRNA Highly expressed in GBC). Coexpression network analysis showed that lncRNA-HGBC was biologically associated with other 17 lncRNAs and 42 proteincoding genes (Additional file 1: Table S4 and Additional file 3: Figure S1B). To further validate the increased level of lncRNA-HGBC in GBC, we examined lncRNA-HGBC expression in another set of 43 cases containing both cancer and adjacent non-tumor tissues, and found that lncRNA-HGBC level was significantly higher in GBC tissues than in benign tissues $(P=0.0074$; Fig. 1a). Next, to determine if lncRNA-HGBC expression level is related to $\mathrm{GBC}$ progression, we analyzed the association between lncRNA-HGBC levels and clinicopathological characteristics in those GBC patients. Using the median expression level of lncRNA-HGBC as a cutoff, 43 GBC patients were stratified into two groups with low and high lncRNA-HGBC expression. As shown in Table 1, statistical analyses showed that high lncRNA-HGBC level was positively correlated with TNM stage $(P=$ $0.0096)$ and lymph node metastasis $(P=0.0191)$. Accordingly, Kaplan-Meier and log-rank tests indicated that high lncRNA-HGBC expression levels were significantly correlated with reduced overall survival (OS) $(P<0.001$, Fig. 1b), implicating an active role in cancer metastasis.

The full length of lncRNA-HGBC was successfully obtained by rapid amplification of the $5^{\prime}$ and $3^{\prime}$ cDNA ends (RACE) assays (Fig. 1c and Additional file 3: Figure $\mathrm{S} 1 \mathrm{C})$. We confirmed the full-length sequence of lncRNA-HGBC by running PCR with 3 pairs of fragmented primers (Additional file 3: Figure S1D). Consistent with the RACE data, Northern blot validated that RNA full length of lncRNA-HGBC was $\sim 2 \mathrm{~kb}$ in length in GBC cell lines (Fig. 1d). To further determine the subcellular localization of lncRNA-HGBC, we separated the nuclear and cytoplasm fraction of NOZ cells and performed qRT-PCR. The results suggested that this lncRNA was mainly located in the cytoplasm (Additional file 3: Figure S1E). Fluorescence in situ hybridization (FISH) analysis indeed revealed this cytoplasm distribution (Fig. 1e). In order for this gene product representing non-protein coding RNA, we employed the sequence analysis program by ORF Finder from the National Center for Biotechnology Information and the result showed that it failed to predict a protein of more than 90 amino acids (Additional file 3: Figure S1F). The coding probability of lncRNA-HGBC was as low as 0.029 if any, as calculated by Coding-Potential Assessment Tool (CPAT) [25] (Additional file 3: Figure S1G). In addition, codon substitution frequency (CSF) analysis indicated that lncRNA-HGBC did not have protein-coding potential (Additional file 3: Figure $\mathrm{S} 1 \mathrm{H}$ ). Furthermore, the fulllength lncRNA-HGBC was lack of the ability to express any protein using in vitro translation assay (Fig. 1f), underscoring that LncRNA-HGBC is a non-protein coding RNA.

\section{LncRNA-HGBC promotes GBC cell proliferation in vitro and in vivo}

Next, we sought to determine effects of lncRNA-HGBC on GBC cell proliferation and tumorigenesis. First, we measured the expression of lncRNA-HGBC in four GBC cell lines and found that NOZ and SGC-996 cells showed higher levels of lncRNA-HGBC than GBC-SD and EH-GB1 (Fig. 2a). Then, we stably silenced lncRNA-HGBC via gene shRNA in NOZ and SGC-996 cell lines (Fig. 2b and Additional file 3: Figure S2A). Knockdown of lncRNA-HGBC in either one of two shRNAs led to significantly decreased cell proliferation over a 5-day culture (Fig. 2c). Consistent 


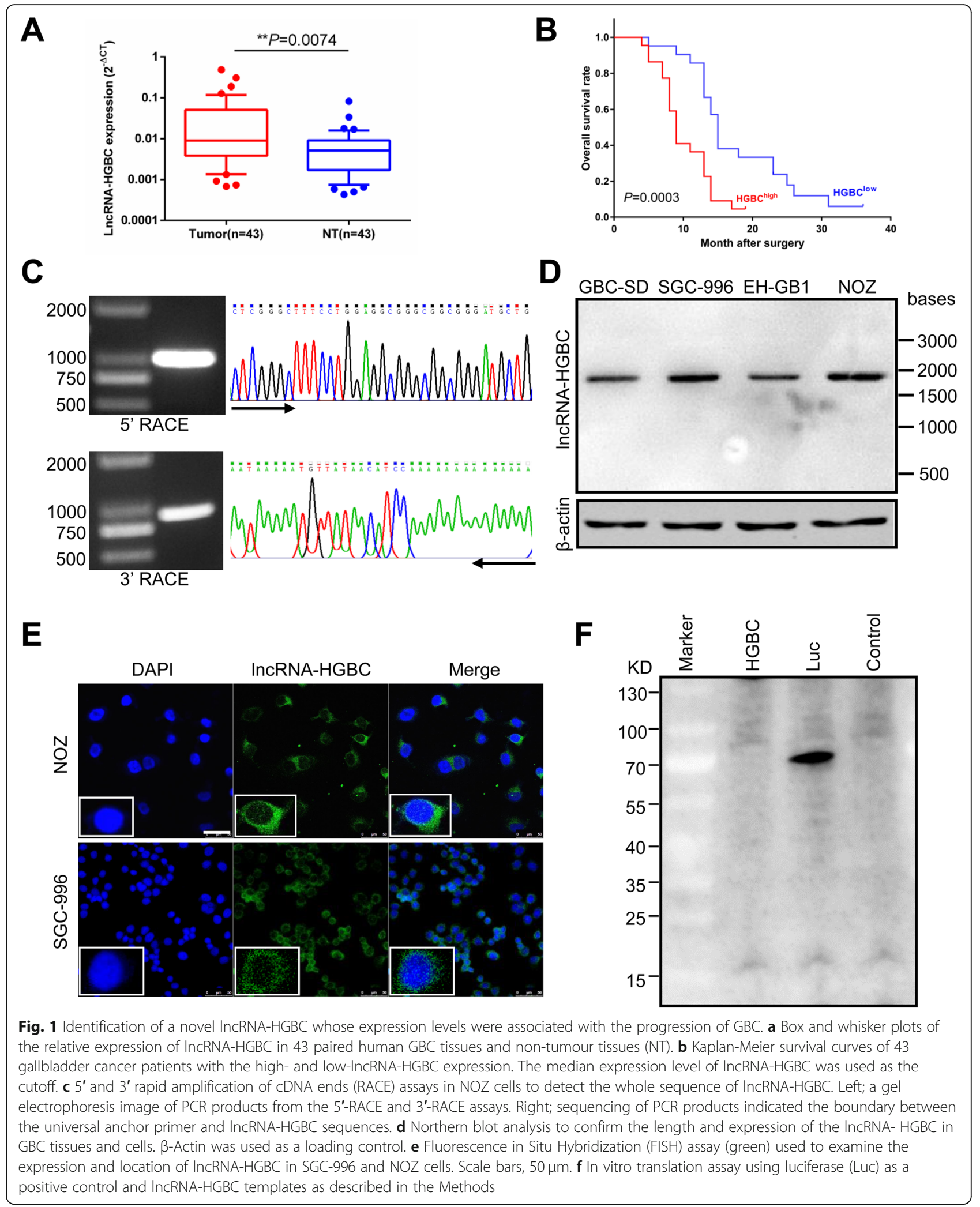

with the proliferation data, silencing lncRNA-HGBC by shRNA1 and shRNA2 also notably decreased the colony formation ability of both cell lines to form colony (Fig. 2d and Additional file 3: Figure S2B). Furthermore, to determine the effects of IncRNA-HGBC on GBC growth in vivo, lncRNA-HGBC-knockdown or control NOZ cells were 
Table 1 Association between IncRNA-HGBC expression and clinicopathologic characteristics of GBC patients in the study cohort

\begin{tabular}{|c|c|c|c|c|c|}
\hline \multirow[t]{2}{*}{ Characteristics } & \multirow[t]{2}{*}{ Cases } & \multicolumn{2}{|c|}{ LncRNA-HGBC expression } & \multirow{2}{*}{$\begin{array}{l}x^{2} \\
\text { value }\end{array}$} & \multirow[t]{2}{*}{$P$ value } \\
\hline & & Low (\%) & High (\%) & & \\
\hline Sex & & & & 0.297 & 0.585 \\
\hline Male & 14 & $6(28.6 \%)$ & $8(36.4 \%)$ & & \\
\hline Female & 29 & $15(71.4 \%)$ & $14(63.6 \%)$ & & \\
\hline Age (years) & & & & 0.009 & 0.924 \\
\hline$<60$ & 12 & $6(28.6 \%)$ & $6(27.3 \%)$ & & \\
\hline$\geq 60$ & 31 & $15(71.4 \%)$ & $16(72.7 \%)$ & & \\
\hline Histology differentiation & & & & 0.007 & 0.933 \\
\hline Well or moderate & 33 & $16(76.2 \%)$ & $17(77.3 \%)$ & & \\
\hline Poor & 10 & $5(23.8 \%)$ & $5(22.7 \%)$ & & \\
\hline TNM stage (AJCC) & & & & 6.702 & 0.0096 \\
\hline $0-11$ & 20 & $14(66.7 \%)$ & $6(27.3 \%)$ & & \\
\hline$I I I-I V$ & 23 & $7(33.3 \%)$ & $16(72.7 \%)$ & & \\
\hline Lymph node Metastasis & & & & 5.495 & 0.0191 \\
\hline Present & 18 & $5(23.8 \%)$ & $13(59.1 \%)$ & & \\
\hline Absent & 25 & $16(76.2 \%)$ & $9(40.9 \%)$ & & \\
\hline
\end{tabular}

AJCC American Joint Committee on Cancer, Bold type indicates statistical significance

injected subcutaneously into nude mice. The results showed that tumor volume and tumor weight in mice injected with lncRNA-HGBC-knockdown NOZ cells were significantly decreased to approximately $30 \%$ of those developed in control mice (Fig. 2e and Additional file 3: Figure $\mathrm{S} 2 \mathrm{C}$ ). Agreed with these data, cell proliferation using proliferating cell nuclear antigen (PCNA) assay unveiled a decreased level of cell proliferation in lncRNA-HGBCdepleted tumors compared with the control group (Fig. 2f).

To further confirm the effect of IncRNA-HGBC on GBC tumorigenesis, we employed a complementary approach by developing cells to stably overexpress lncRNA-HGBC in GBC-SD and EH-GB1 cells that express low levels of endogenous lncRNA-HGBC (Fig. 2g and Additional file 3: Figure S2D). As expected, LncRNA-HGBC-overexpressing cells (Lv-HGBC) showed increased cell proliferation and cell colonies compared with empty vector-transfected cells (Lvcontrol) (Fig. 2h, i and Additional file 3: Figure S2E). In addition, tumor volume and weight were 2.5 -fold greater in the IncRNA-HGBC-overexpressing group than those in the controls (Fig. $2 j$ and Additional file 3: Figure S2F). IHC staining showed that PCNA was upregulated in IncRNAHGBC-overexpressing xenograft tumor tissues (Fig. 2k). Collectively, our data demonstrate that lncRNA-HGBC acts as a tumor-promoting factor to enhance tumorigenesis of GBC cells.

\section{LncRNA-HGBC drives GBC cells to undergo EMT and cancer metastasis}

Given that the lncRNA-HGBC RNA level was positively related to the existence of lymph node metastasis, we reasonably postulate that lncRNA-HGBC augments GBC cell invasive behavior. To test the hypothesis, we exploited cell transwell migration and matrigel invasion assays, and found that knocking down endogenous IncRNA-HGBC by specific shRNAs dramatically reduced the cell migration and invasion by around $30-40 \%$ of controls in NOZ and SGC-996 cells (Fig. 3a, b and Additional file 3: Figure S3A, B). Conversely, overexpression of lncRNA-HGBC in GBC-SD and EH-GB1 cells increased the migration and invasion by $20-50 \%$ (Fig. 3c, $\mathrm{d}$ and Additional file 3: Figure S3C, D). It is well established that increased metastatic capability of GBC is intimately associated with tumor cell phenotypic transformation, an event termed epithelial-mesenchymal transition (EMT) [26]. To evaluate the possibility of EMT involved in the invasiveness of GBC, we monitored EMT-specific markers such as vimentin and $\mathrm{N}$-cadherin. lncRNA-HGBC knockdown in NOZ and SGC-996 cells decreased expression of vimentin and $\mathrm{N}$-cadherin (Fig. $3 e)$. On the contrary, IncRNA-HGBC overexpression in GBC-SD and EH-GB1 led to increased both levels (Fig. 3f). To confirm these findings in vitro and visualize the likelihood of acquired tumor metastasis in vivo, we established a liver metastasis tumour model in nude mice by injecting NOZ cells to the spleen. Five out of the six mice (5/6) in the control group showed increased luciferase signals and intrahepatic metastatic nodules in their livers after 6 week-transplantation, whereas only three of the six mice (3/6) injected with IncRNA-HGBCshRNA NOZ cells developed liver nodules. Strikingly, metastatic foci seen in the liver in lncRNA-HGBC- 


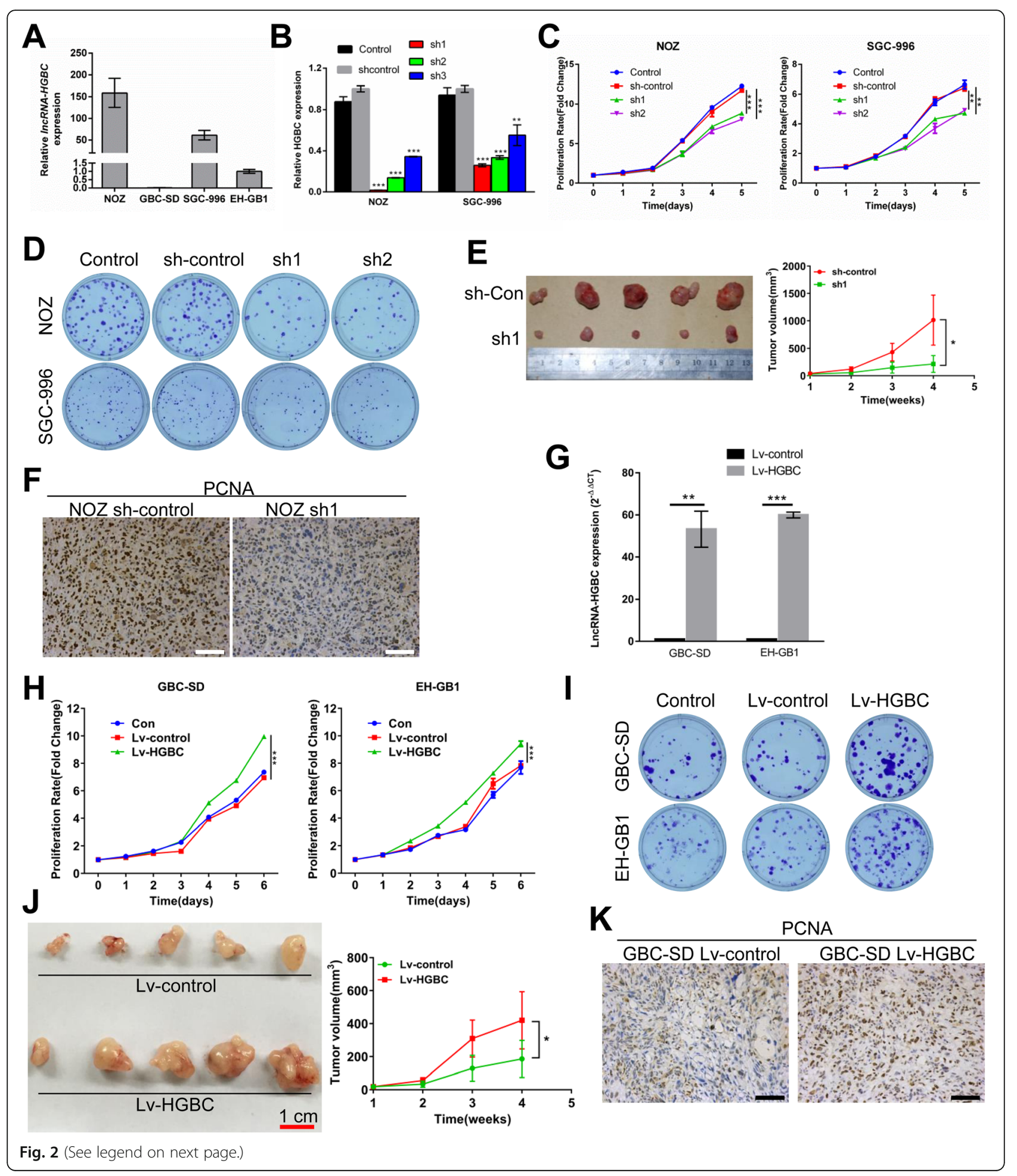




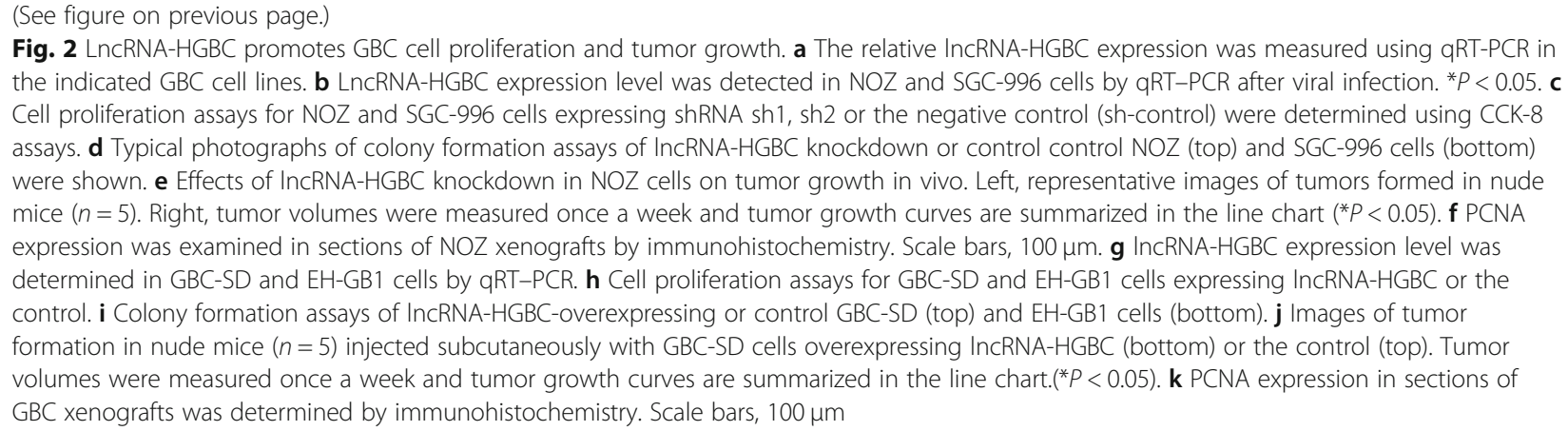

shRNA tumor mice was as low as $10 \%$ of control metastatic tumors (Fig. 3g, h). Taken together, these data strongly suggest that lncRNA-HGBC commits GBC cells to undergoing EMT and promoting tumor metastasis.

\section{HuR physically interacts with and stabilizes IncRNA-HGBC} Growing evidence has pointed to the notion that many lncRNAs can function to regulate some target gene expression through direct interaction with proteins [27-29]. To explore the potential binding proteins for lncRNA-HGBC in GBC cells, we performed a biotin-labeled RNA pulldown assay followed by silver staining (Fig. 4a). A protein band specifically presented in lncRNA-HGBC was located at approximately $37 \mathrm{kD}$ and then was subjected to sequence analysis via mass spectrometry. With great interest in the analyzed data (Additional file 1: Table S5), we paid particular attention to an RNA-binding candidate HuR that was confidence score $>100$ and molecular weight (MW) 30-40 $\mathrm{KD}$. Western blot assay confirmed that HuR was a specific binding protein for lncRNA-HGBC (Fig. 4b). An RNA immunoprecipitation (RIP) assay was further utilized to validate the specific interaction between IncRNA-HGBC and $\mathrm{HuR}$, as compared with the positive control EIF4E mRNA [30] that is enriched in HuR binding (Fig. 4c). Next, to interrogate which specific region within IncRNA-HGBC contributes to $\mathrm{HuR}$ binding, we constructed four different deletion fragments of lncRNA-HGBC based on the secondary structure of lncRNA-HGBC that was predicted from RNA fold Web server (http://rna.tbi.univie.ac.at/cgi-bin/ RNAfold.cgi) (Fig. 4d). Subsequently, RNA pulldown assay followed by WB showed HuR specific binding sequence was located within 1759-1906 nt-long region that harbors HuR-binding motif (UUUUUUGUUUUGGCAAAUAGUU AUUUUUCAUU) (Fig. 4e), indicating that 1759-1906 nt renders lncRNA-HGBC able to bind to HuR.

To investigate possible effects of IncRNA-HGBC on HuR expression, we measured protein expression of $\mathrm{HuR}$ in IncRNA-HGBC-overexpressing GBC-SD and lncRNA-HGBC-knockdown NOZ cells. The results showed that IncRNA-HGBC did not have ability to change HuR expression (Additional file 3: Figure S4).
Given the rigorous activity of $\mathrm{HuR}$ in stabilizing mRNAs and lncRNAs [31, 32], we examined whether HuR affects the stability of lncRNA-HGBC in GBC cells. As shown in Fig. 4f, the expression of lncRNA-HGBC in NOZ cells was reduced by 65.8 and $32.5 \%$ in two independent HuR-knockdown cells, respectively. To further investigate if this reduction was due to increased lncRNAHGBC decay, we incubated NOZ cells with $\alpha$-amanitin to block de novo RNA transcription and then measured the expression of IncRNA-HGBC over a $30 \mathrm{~h}$ period. The depletion of HuR decreased the half-life of lncRNAHGBC level from $18 \mathrm{~h}$ to $5 \mathrm{~h}$ (Fig. $4 \mathrm{~g}$ ), indicating that $\mathrm{HuR}$ contributed to stabilizing lncRNA-HGBC. Collectively, these data strongly suggest that $\mathrm{HuR}$ interacts with and stabilizes lncRNA-HGBC.

\section{LncRNA-HGBC functions as a competing endogenous RNA} by directly binding to and inhibiting miR-502-3p

Emerging lines of evidence have reported that cytoplasm lncRNAs can function as competing endogenous RNAs (ceRNAs) by binding to and sequestering specific miRNAs that block target gene expression [19, 20]. Given that lncRNA-HGBC is mainly located in the cytoplasm, we hypothesized that lncRNA-HGBC may function as miRNA sponge to restore gene expression targeted by miRNA in GBC progression. In a set of miRNAs that are putatively bound to lncRNA-HGBC in the Segal Lab program (Eran Segal; http://132.77.150.113/pubs/mir07/ mir07_prediction.html) [24] (Additional file 1: Table S6), we were particularly interested in six tumor suppressorassociated miRNAs including miR-1, miR-26a, miR-630, miR-122, miR-502-3p and miR-618. To obtain the bona fide IncRNA-miRNA interaction, we subcloned fulllength lncRNA-HGBC into the pmirGLO dual luciferase reporter vector. Dual luciferase assay showed that miR502-3p and miR-618 could suppress the luciferase activity of lncRNA-HGBC, but not other four miRNAs (Fig. 5a), indicating a possible interaction between IncRNA-HGBC and miR-502-3p or miR-618. We unexpectedly found miR-502-3p was indeed downregulated in GBC tissues compared with adjacent non-tumor 

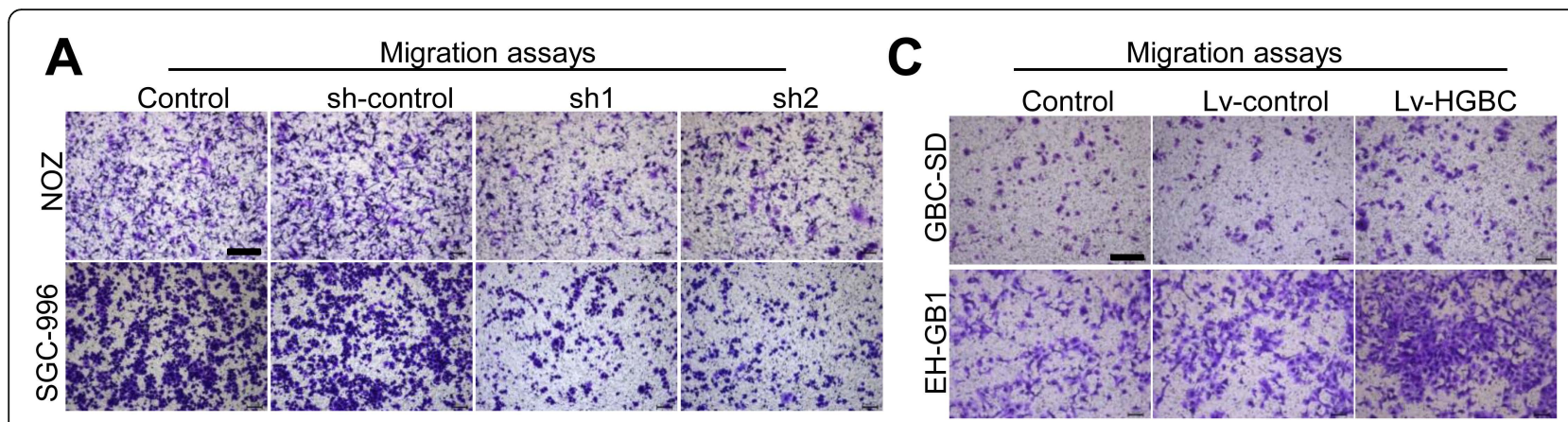

\section{B}

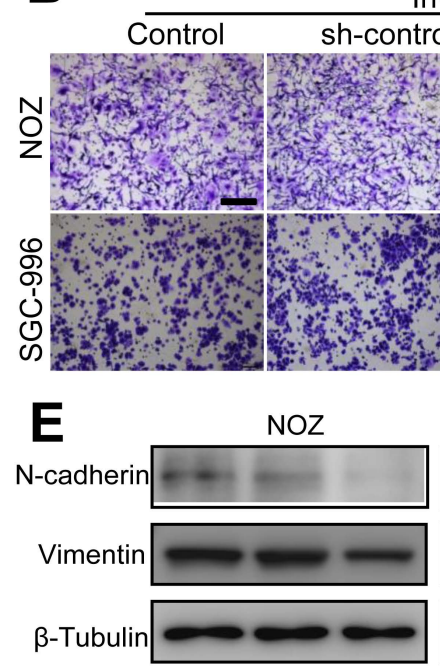

Invasion assays
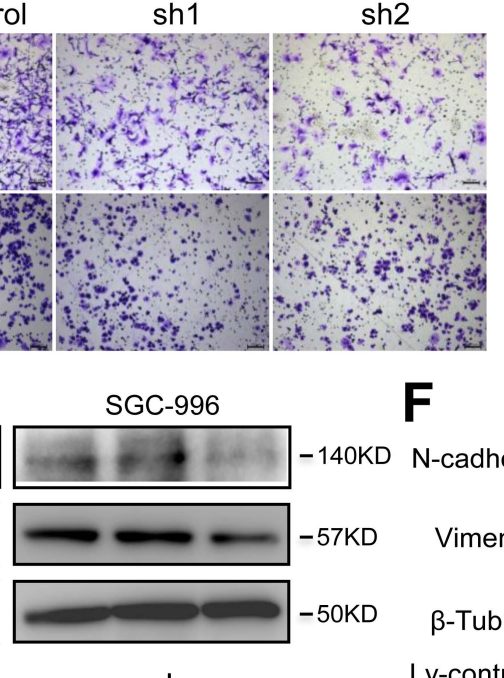

D

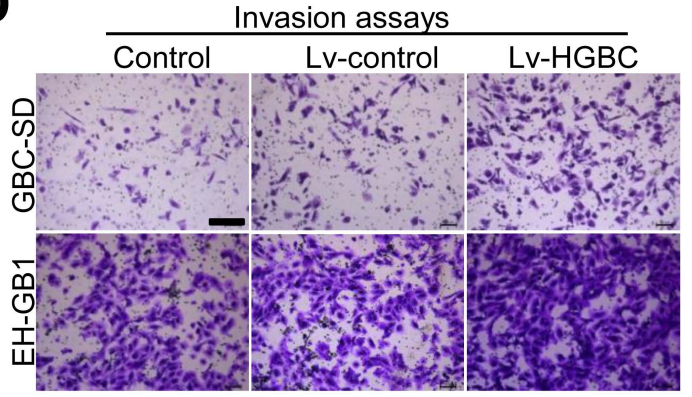

$\mathbf{F}$

GBC-SD

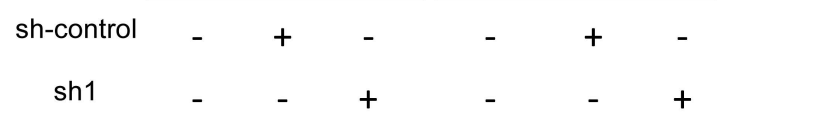

$\mathrm{N}$-cadherin

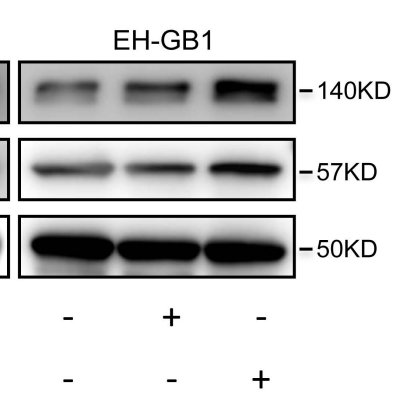

\section{G}
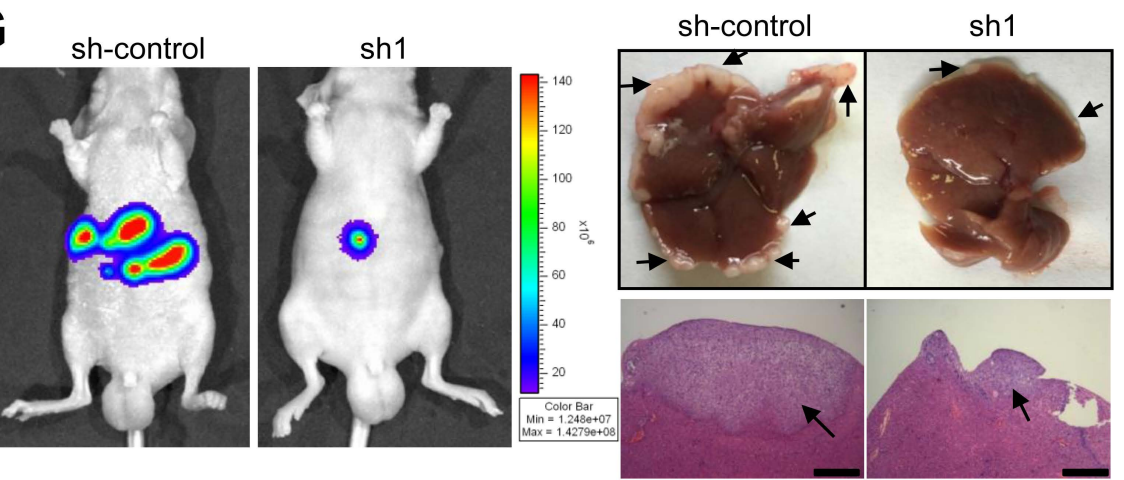

$\mathrm{H}$

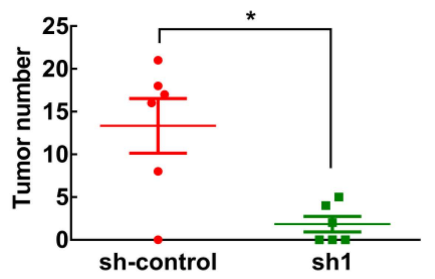

Fig. 3 LncRNA-HGBC reinforces the invasive capacity of GBC cells. a, b Transwell assays (a) and Invasion assays (b) were used in NOZ and SGC996 cells. Scale bars, $200 \mu \mathrm{m}$. c, d Transwell assays (c) and Invasion assays (d) were used in InCRNA-HGBC-overexpressing GBC-SD and EH-GB1 cells. Scale bars, 200 mm. e, f The protein levels of N-cadherin and Vimentin in control and IncRNA-HGBC-knockdown NOZ (left) or SGC-996 (right) cells, and in control and InCRNA-HGBC-overexpressing GBC-SD (left) or EH-GB1 (right) cells. g Representative images of luciferase signals in mice at the 6 weeks after intrasplenic injection with NOZ cell clones (left). Representative livers were shown and the isolated liver tissues sections were stained by hematoxylin and eosin (righ). Arrows indicate the metastasis nodules. Scale bars, $500 \mu \mathrm{m}$. $\mathbf{h}$ The average number of liver metastases in the intrasplenic injection model. Data are presented as mean \pm SD of three independent experiments. ${ }^{*} P<0.05,{ }^{* *} P<0.01,{ }^{* * *} P<0.001$ 


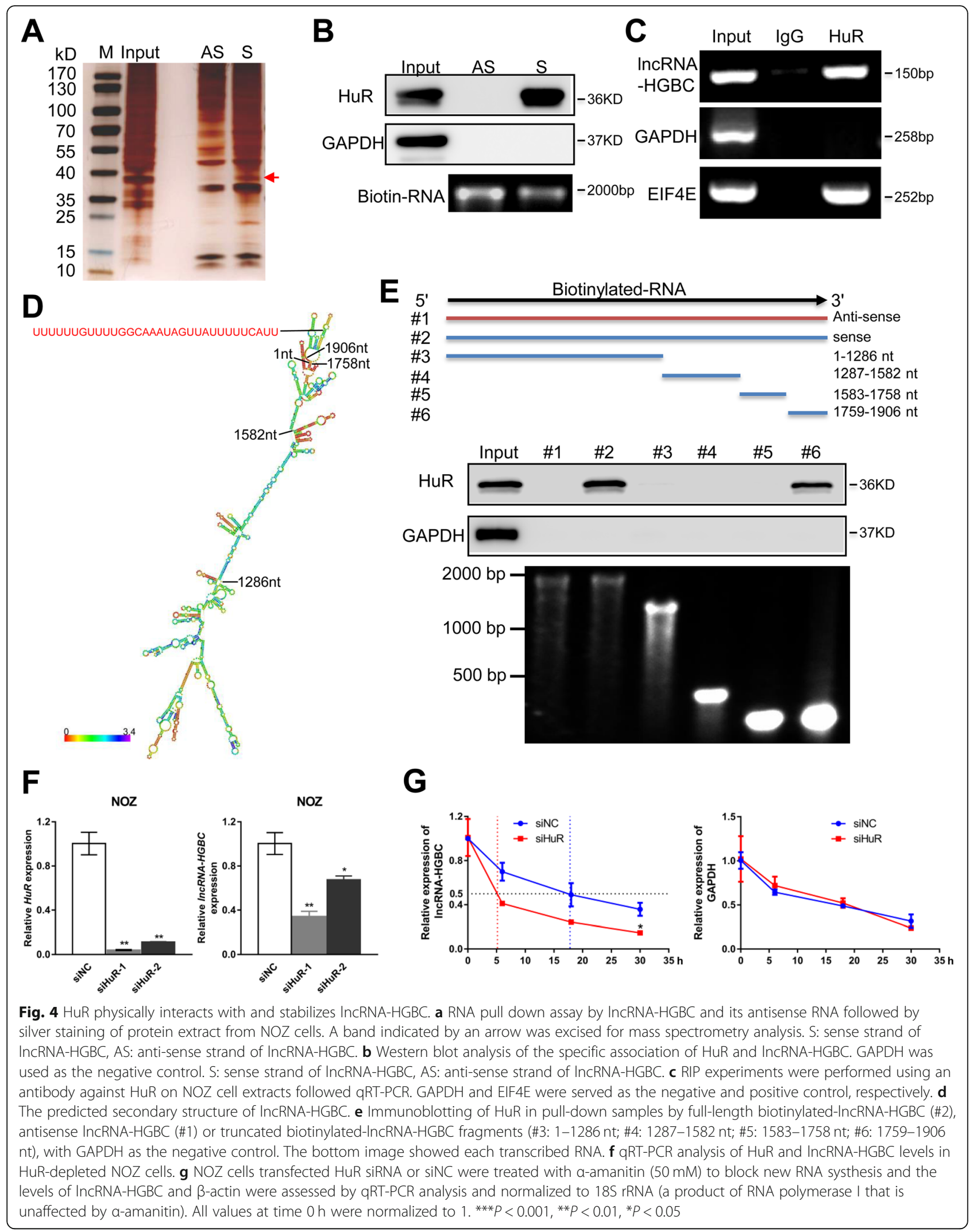




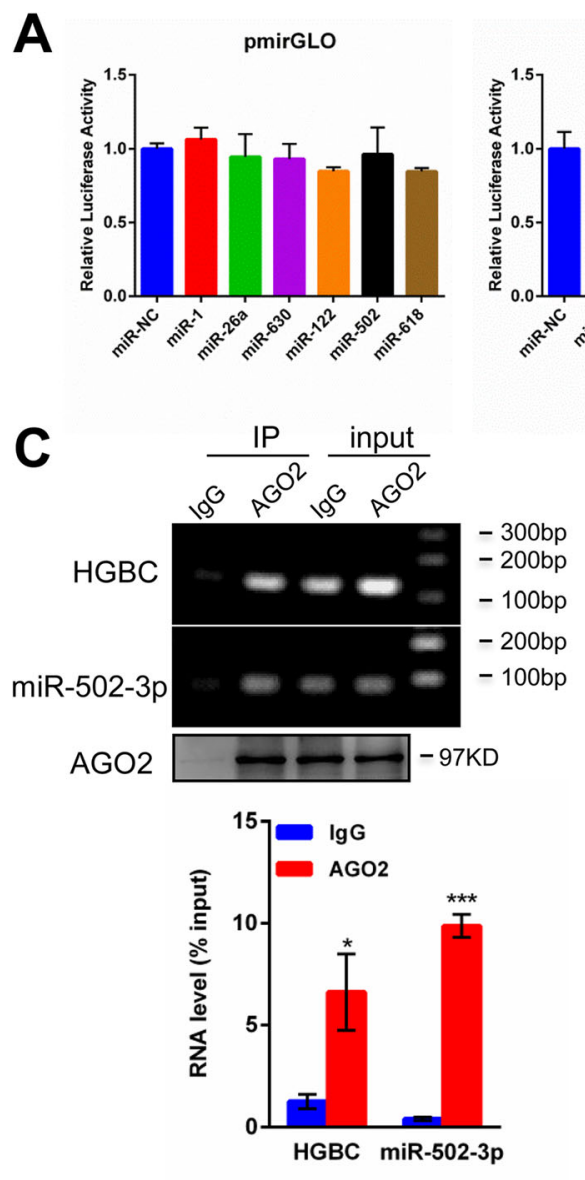

E

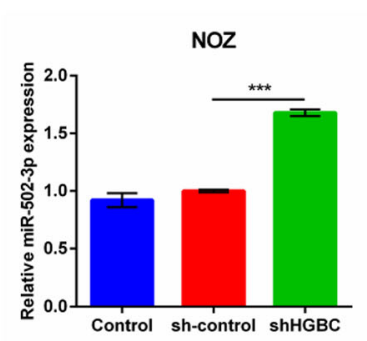

pmirGLO-HGBC

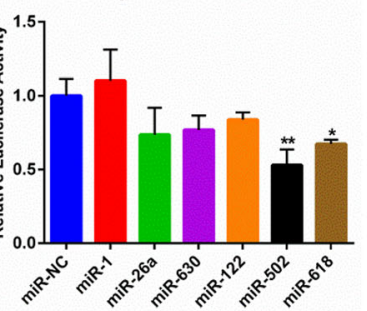

BIICRNA-HGBCWT 5 5' ...GCTCGCATGGTAGGGTGCATg... $3^{\prime}(1176-1183)$

miR-502-3p 3' ACUUAGGAACGGGUCCACGUAA 5'

InCRNA-HGBC 502-3PMUT 5' ...GCTCGCATGGTA-........ g... 3'

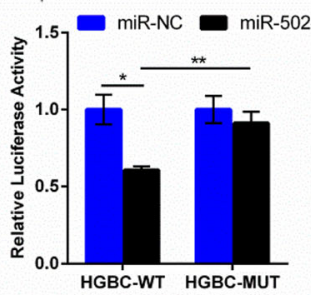

D
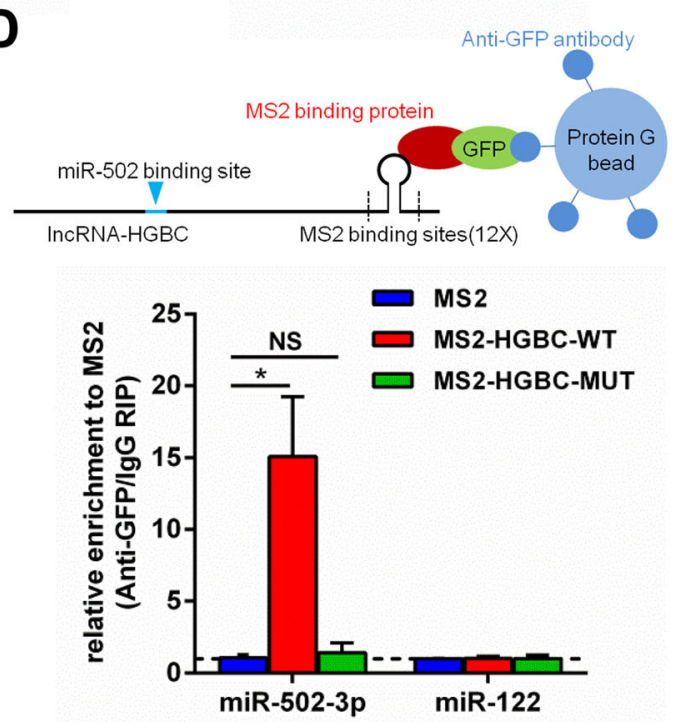

$\mathbf{F}$

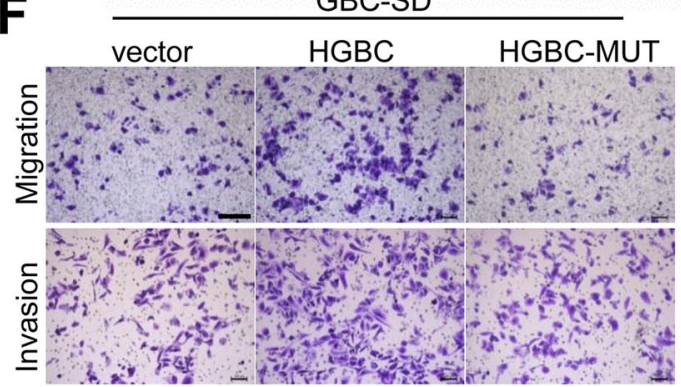

EH-GB1

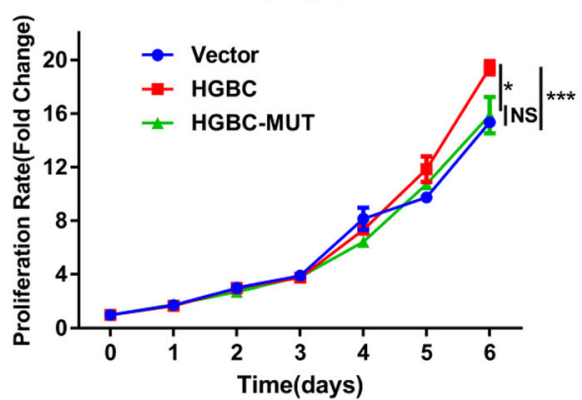

Fig. 5 (See legend on next page.) 


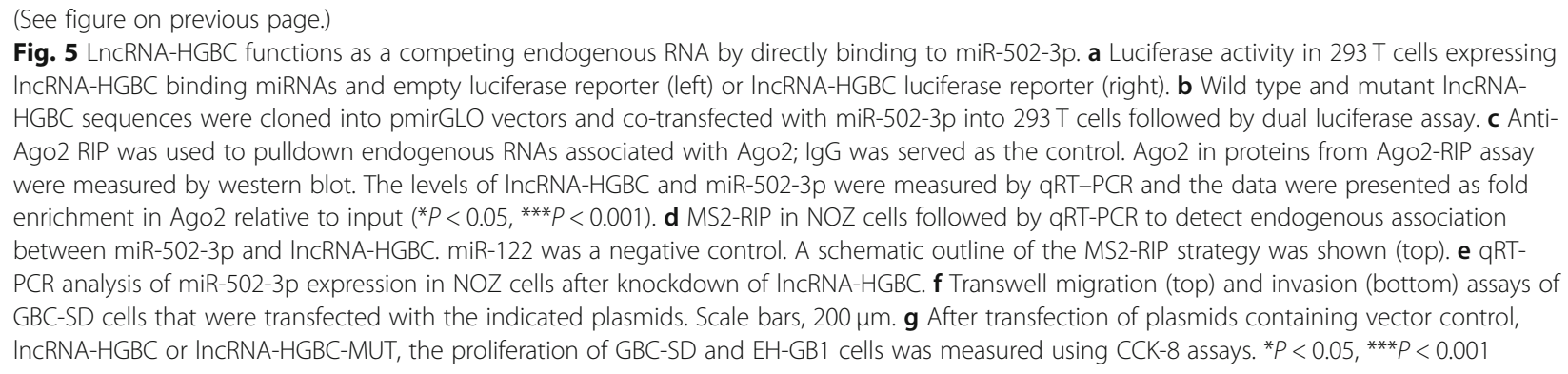

tissues based on our previous miRNA microarray results [33] (data not shown, GEO accession number GSE90001). Therefore, we were primarily focused on the interaction between IncRNA-HGBC and miR-502-3p. Once miR-502-3p binding motif of GGTGCAT between 1176 and $1183 \mathrm{nt}$ of lncRNA-HGBC was deleted as mutant HGBC-MUT, luciferase activity was not suppressed, as compared with decreased activity in the presence of wild type of miR-502-3p binding motif (Fig. 5b). A large body of mechanistic studies focusing on the interaction between miRNA and targeted mRNA have established the notion that miRNAs bind to their mRNA targets and cause translational suppression and/or RNA degradation by forming a complex with Argonaute2 (AGO2) [34]. To test this action model, RNA immunoprecipitation (RIP) experiments were employed in NOZ cell extracts using an AGO2 antibody. As shown in Fig. 5c, both lncRNAHGBC and miR-502-3p were specifically enriched in AGO2 antibody-associated complex, but not in the control IgG, suggesting that miR-502-3p is a bona fide lncRNA-HGBC-targeting miRNA. To further identify whether lncRNA-HGBC directly binds to endogenous miR-502-3p, we performed MS2-RIP to pulldown endogenous miRNAs associated with lncRNA-HGBC (Fig. $5 \mathrm{~d}$, above). The results demonstrated that IncRNAHGBC in NOZ cells was significantly associated with miR-502-3p, but not with irrelevant microRNA (miR122). However, mutation of miR-502-3p binding site in lncRNA-HGBC abolished their association (Fig. 5d, below), supporting the direct interaction between IncRNA-HGBC and miR-502-3p.

To further clarify the regulatory relationship of gene expression between IncRNA-HGBC and miR-502-3p, we evaluated miR-502-3p levels in cells expressing divergent levels of lncRNA-HGBC. qRT-PCR results indicated that miR-502-3p was markedly upregulated by $60 \%$ after lncRNA-HGBC knockdown in NOZ cells (Fig. 5e, left). Conversely, the expression of miR-502-3p was inhibited to $50 \%$ of control after lncRNA-HGBC overexpression in EH-GB1 cells (Fig. 5e, right). However, the expression of lncRNA-HGBC was not altered after miR-502-3p overexpression or knockdown (Additional file 3: Figure S5A), suggesting that lncRNA-HGBC acts as a sponge to inhibit
miR-502-3p, but did not induce degradation. According to the ceRNA feature that the expression level of lncRNAs should be comparable to their binding miRNAs, we found that the expression levels of IncRNA-HGBC and miR502-3p in NOZ cells were approximately 382 and 450 copies per cell, respectively. To examine whether the activity of lncRNA-HGBC depends on its binding to miR502-3p, cck-8 and Transwell assays were performed after ectopic expression of wild type lncRNA-HGBC and binding mutant HGBC-MUT. The results showed that enforced expression of IncRNA-HGBC, but not HGBCMUT, significantly promoted cell migration, invasion and proliferation (Fig. 5f-g). As wild type lncRNA-HGBC was knocked down, the opposite effects were observed (Additional file 3: Figure S5B-S5D). Collectively, these data demonstrate that lncRNA-HGBC physically binds to miR502-3p and may serve as a sponge to inhibit miR-502-3p.

\section{LncRNA-HGBC upregulates expression of SET via sequestration of miR-502-3p}

miR-502-3p is appreciated to target multiple proteincoding genes including SET [35] that plays an important role in the development of various carcinomas [36, 37]. More importantly, SET was also upregulated in GBC tissues in our previous microarray data [23] (data not shown, GEO accession number: GSE76633). To decipher the regulatory mechanisms of miR-502-3p on SET, we transfected a luciferase reporter vector harboring 3' UTR of SET into $293 \mathrm{~T}$ cellsand luciferase activity was then evaluated in the transfection of miR-502-3p mimics. As compared to the control vector, miR-502-3p mimics significantly reduced the luciferase activity of the SET reporter vector (Additional file 3: Figure S6A). Furthermore, after overexpression or knockdown of miR-502-3p in GBC cells (Additional file 3: Figure S6B), the expression of SET was decreased by miR-502-3p or increased by antimiR-502-3p, respectively, at both mRNA and protein levels (Additional file 3: Figure S6C, S6D). These data indicate that SET is a direct target of miR-502-3p.

Since lncRNA-HGBC shares miR-502-3p binding with 3' UTR of SET, we wondered whether lncRNA-HGBC modulates miR-502-3p-mediated inhibition of SET in GBC cells. First, we examined whether lncRNA-HGBC has the ability 
to inhibit miR-502-3p activity. A pmirGLO-SET luciferase reporter vector was co-transfected with lncRNA-HGBC overexpression plasmid, miRNA mimic NC and/or miR502-3p mimic into GBC-SD cells. As shown in Fig. 6a, luciferase activity of the SET reporter vector was inhibited by overexpressing miR-502-3p but was induced by lncRNAHGBC overexpression. Once both miR-502-3p and lncRNA-HGBC were introduced, the luciferase activity was restored to the control level, implying that lncRNA-HGBC has the ability to release SET from miR-502-3p inhibition by sequestering miR-502-3p. To provide direct evidence for regulation of SET mRNA and protein expression, we introduced lncRNA-HGBC shRNA and miR-502-3p inhibitor into NOZ cells that express high endogenous levels of lncRNA-HGBC. The results showed that lncRNA-HGBC knockdown suppressed SET expression, but the addition of miR-502-3p inhibitor increased the SET expression to the control levels (Fig. 6b, left and Additional file 3: Figure S6E, left). On the contrary, in EH-GB1 cells that express low endogenous levels of IncRNA-HGBC, overexpression of lncRNA-HGBC induced SET expression; however, the additional introduction of miR-502-3p decreased SET to the basal level (Fig. 6b, right and Additional file 3: Figure S6E, right). Accordingly, inhibition of miR-502-3p sufficiently reversed lncRNA-HGBC shRNA-inhibited cell proliferation, migration and invasion in NOZ cells (Fig. 6c, d and Additional file 3: Figure S6F). In contrast, miR-502-3p overexpression ablated increased cell growth, migration and invasion induced by lncRNA-HGBC overexpression in EHGB1 cells (Fig. 6c, e and Additional file 3: Figure S6F). To examine whether IncRNA-HGBC-induced SET expression depends on its binding to miR-502-3p, overexpression of lncRNA-HGBC in both GBC-SD and EH-GB1 cells resulted in increases in SET expression at mRNA and protein levels, whereas miR-502-3p-binding mutation of lncRNA-HGBC failed to induce SET expression. (Fig. 6f, g and Additional file 3: Figure S6G). In addition, the expression pattern of SET was similar to the expression of lncRNA-HGBC in the four $\mathrm{GBC}$ cell lines (Additional file 3: Figure S6H). Consistent with these data, IHC analysis revealed that SET expression was decreased in lncRNA-HGBC-depleted xenograft tumors, but increased in lncRNA-HGBC-overexpressing xenograft tumors, compared with the control group (Additional file 3: Figure S6I), suggesting that there was a coexpression relationship between IncRNA-HGBC and SET. In summary, these data strongly suggest that lncRNAHGBC regulates SET expression by competitively binding to and inhibiting miR-502-3p.

AKT is the downstream effector of SET and relationship of IncRNA with, miR-502-3p, SET and p-AKT in GBC

We next examined the potential molecular mechanisms of miR-502-3p and SET involved in GBC metastasis. As shown in Additional file 3: Figure S7A-S7E, miR-502-3p strongly inhibited the proliferation, colony formation and invasion capabilities of all of four GBC cell lines. Agreed with these data, direct knockdown of SET dramatically inhibited GBC cell proliferation and invasion (Additional file 3: Figure S8A-S8D). There is accumulating research evidence demonstrating that SET-induced oncogenic activity in various cancers is dependent on activation of AKT $[36,37]$. Thus, it is reasonable to postulate that the axis of lncRNA-HGBC-miR-502-3p-SET-AKT acts as a pivotal system to mediate GBC development and metastasis. To approve this activated signaling in our system, we first examined AKT activation. Western blot assays showed that lncRNA-HGBC knockdown in NOZ and SGC-996 cells significantly inhibited the activated AKT (Fig. 7a); however, overexpression of lncRNA-HGBC induced phosphorylation of AKT in GBC-SD and EH-GB1 cells (Fig. 7b).

To determine if the lncRNA-HGBC-induced AKT activation is dependent on participation of miR-502-3p, we used miR-502-3p binding mutation of lncRNA-HGBC and found that lncRNA-HGBC overexpression in GSCSD and EH-GB1 cells led to increases in AKT phosphorylation, N-cadherin, and vimentin, whereas lncRNA-HGBC MUT failed to induce these protein levels (Fig. 7c). In addition, introduction of miR-502-3p to lncRNA-HGBCoverexpressing EH-GB1 cells decreased these protein expressions; however, inhibition of miR-502-3p restored these levels that were inhibited by shHGBC in NOZ cells (Fig. 7d). AKT inhibitor MK2206 reversed effects of lncRNA-HGBC on expression of $\mathrm{N}$-cadherin and vimentin (Fig. 7e), suggesting the tumor promoting role of lncRNA-HGBC requires AKT activation.

Finally, to validate the imitate association of individual factors in the IncRNA-HGBC-miR-502-3p-SET-AKT axis in GBC, we analyzed their correlations in 43 pairs of GBC tissue. LncRNA-HGBC was inversely correlated with miR-502-3p, whereas positively correlated with SET and HuR mRNA levels (Fig. 7f). HuR was upregulated in GBC tissues in comparison to non-tumor tissues (Additional file 3: Figure S9). IHC analysis of SET or p-AKT expression showed that both levels were significantly upregulated in GBC specimens compared with those in the non-tumorous tissues (Fig. 7g). Moreover, lncRNAHGBC expression was positively correlated with SET and p-AKT expression (Fig. 7h, i). Collectively, all of these observations indicate that IncRNA-HGBC functions to sequester miR-502-3p then to activate SET and AKT downstream pathway, thus rendering tumor cells highly aggressive.

\section{Discussion}

The human genome is pervasively transcribed to give rise to more than $80 \%$ non-coding genes in which lncRNAs constitute the primary elements, while only about $2 \%$ of 


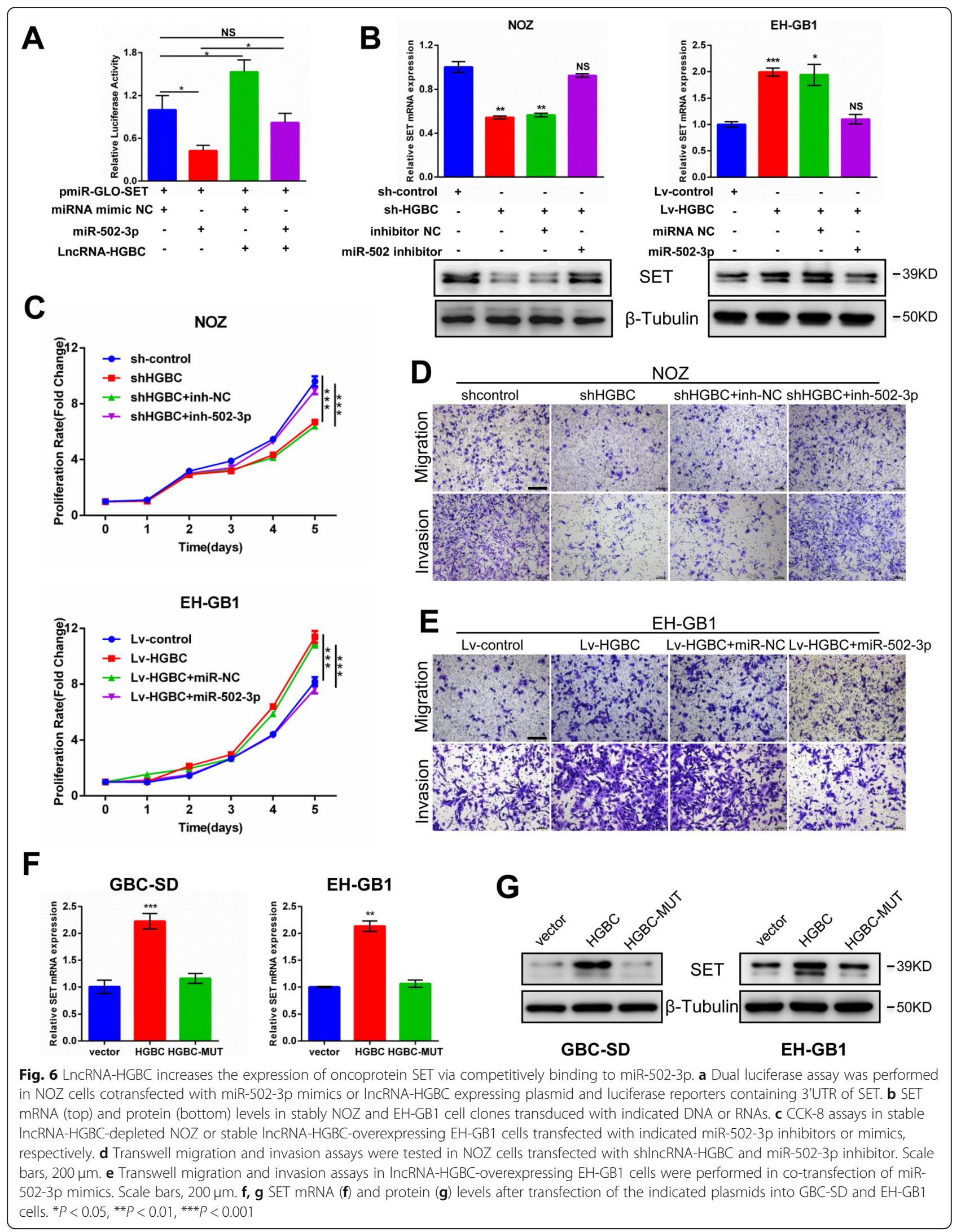




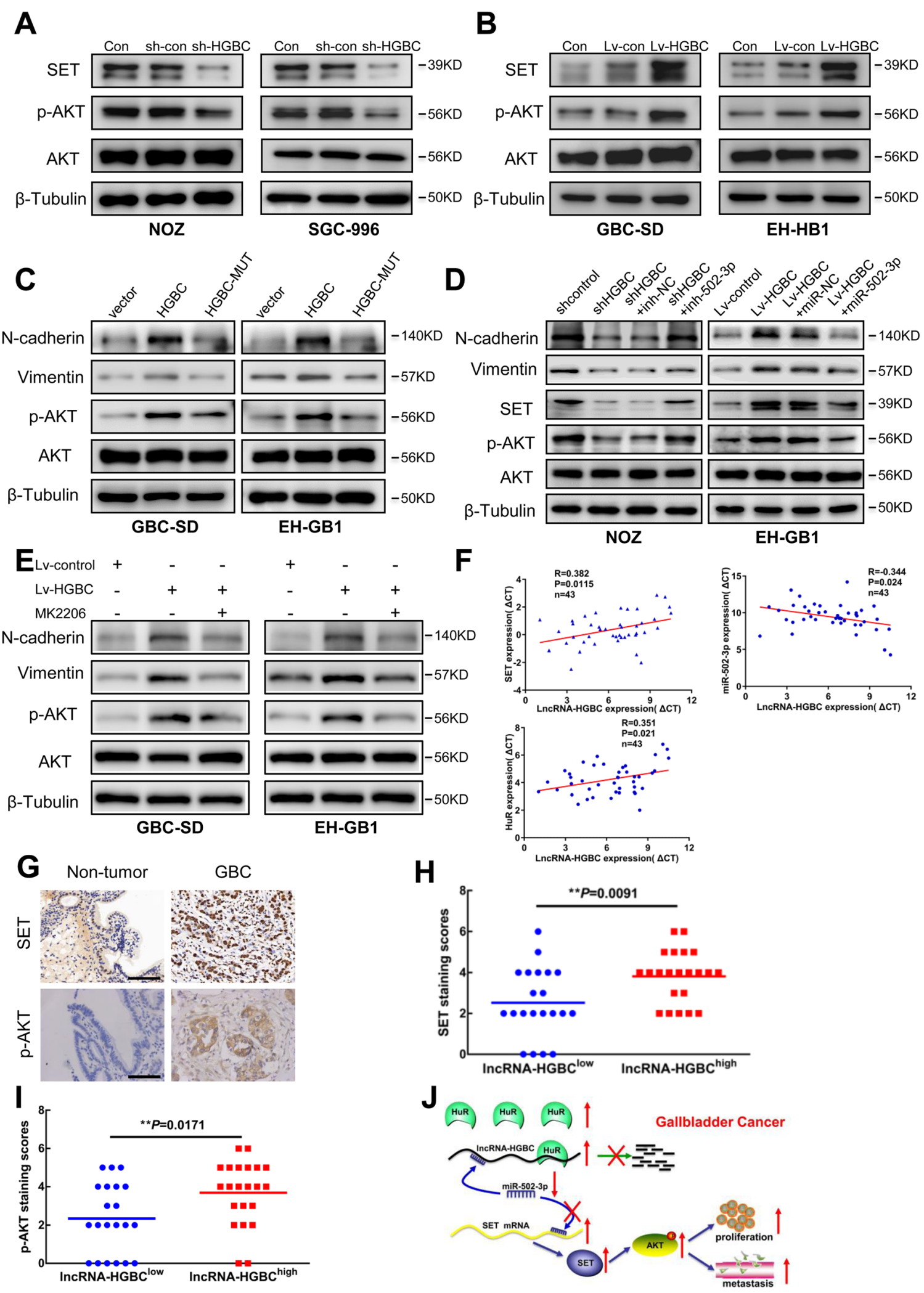

Fig. 7 (See legend on next page.) 
(See figure on previous page.)

Fig. 7 AKT is the downstream effector of SET and relationship of IncRNA with, miR-502-3p, SET and p-AKT in GBC. a Western blot assays of SET, p-AKT and AKT expression in shcontrol- or sh-HGBC-expressing NOZ or SGC-996 cells. b Western blot assays of SET, p-AKT and AKT expression in pcDNA3.1-based vector control and IncRNA-HGBC-expressing GBC-SD and EH-GB1 cells. c Western blotting analysis of N-cadherin, Vimentin, pAKT and AKT expression in GBC-SD (left) and EH-GB1 (right) cells. d Western blotting analysis of N-cadherin, Vimentin, SET, p-AKT and AKT expression in indicated cells. e The expression of N-cadherin, vimentin, phosphorylated AKT and AKT was determined in GBC-SD and EH-GB1 cells in presence or absence of 10 uM MK2206. tubulin was used as the loading control. $\mathbf{f}$ The correlation between IncRNA-HGBC and SET (upper left), miR-502-3p (upper right) or HuR (bottom) expression was detected in 43 GBC specimens by qRT-PCR. The $\Delta C t$ values were subjected to Pearson correlation analysis. $\mathbf{g}$ Representative images of SET (top) or P-AKT (bottom) expression by immunohistochemical staining from non-tumor tissues and GBC tumors. Scale bars, $100 \mu \mathrm{m}$. h-i Scatterplots of the average staining scores for SET (h) or p-AKT (i) expression in GBC patients with low or high expression of IncRNA-HBC. $\mathbf{j}$ Schematic diagram of IncRNA-HGBC functions to promote tumor growth and metastasis in GBC cells

genes encode for proteins. A wealth of research evidence has established the paradigm that lncRNAs are biologically functional molecules, in sharp contrast to initially accepted transcriptional "noise" [14, 38]. Given the cellular distribution of lncRNAs in both nucleus and cytoplasm, lncRNAs utilize a variety of molecular mechanisms to regulate gene activity and protein function, as some of lncRNAs participate in transcriptional interference, RNA splicing and miRNAquenching, while others can directly interact with transcriptional factors, hormone receptors and other RNA-binding proteins [15]. Thereof, it is not surprising to anticipate that deregulation of lncRNAs may result in multiple aspects of cellular dysfunction, even malignant transformation. In the current study, we discovered a novel cytosol lncRNA-HGBC with $1906 \mathrm{nt}$ whose expression level was 16.6-fold higher in GBC than adjacent benign tissue and was correlated with positive lymph node metastasis and poorer patient survival, suggesting that IncRNA-HGBC may be a potential prognostic predictor or therapeutic target for GBC.

It is emerging that one of molecular mechanisms by which lncRNAs regulate gene expression is to interact with miRNA as ceRNAs that bind to MREs and protect miRNAs from binding to and repressing target RNAs [17, 39]. For example, IncRNA- MCM3AP-AS1 promotes tumor growth by specific binding to miR-194-5p, thus sequestering the miRNA and inducing FOXA1 expression [40]. In addition to the capability of MRE binding, lncRNAs display the strong ability to expedite degradation of miRNAs that inhibit target gene expression. IncRNA HOXA11-AS acts as a decoy for miR-1297, resulting in elevated expression of EZH2 in gastric cancer [41]. In concert with these findings, our current study demonstrates that lncRNA-HGBC interacts with miR-502-3p that is a tumor suppressor in hepatocellular carcinoma [35]. In addition, overexpression or knockdown of lncRNA-HGBC can correspondingly alter miR-502-3p expression, but the latter was unable to alter the former expression, indicating that lncRNA-HGBC not only interacts with and removes miR-502-3p from target gene inhibition, but also suppresses miR-502-3p expression. The substantial mechanisms of lncRNA-HGBC for regulating miR-502-3p expression warrant further investigation. Of note, miR-502-3p was down-regulated in GBC tissues and acted as a tumor suppressor to inhibit a target gene SET that promotes tumorigenesis in hepatocellular carcinoma [36], lung cancer [42] and breast cancer [43]. Indeed, lncRNA-HGBC-induced SET expression is fully dependent on the reduced expression or activity of miR-502-3p, because miR-502-3p mimic or overexpression of miR-502-3p can ablate lncRNA-HGBC activity. SET is known to inhibit protein phosphatase 2A (PP2A) [44] that can augment dephosphorylation and inactivationof AKT [45]. Given the feature that IncRNA-HGBC binds to and sequesters multiple miRNAs (e.g. miR-618), not limited to miR-502-3p, it is worthwhile to add considerable effort to reveal additional miRNAs that potentially regulate SET and others during GBC metastasis. The cell proliferation induced by lncRNAHGBC in cultured cells was not as noticeable as developed tumor in vivo. The inconsistence could be ascribed to the following reasons. First, we used CCK8 as an in vitro assay to determine cell proliferation. This assay may have sensitivity limit as it determines cell growth by an enzymemediated colorimetric assay, instead of measuring DNA synthesis with radioactive material. Second, the in vitro changes in cell proliferation are only for tumor cells grown in a well in the absence of other cells or factors. In contrast, the in vivo tumors involve tumor microenvironment including stromal cells, growth factors, extracellular matrix/ proteins and vessels, not limited to tumor cells. For example, lncRNA-HGBC expressed in tumor cells induces other angiogenic factors to stimulate blood vessel development which in turn promotes tumor cell proliferation. Last, we only observed in vitro a few days which do not represent a long period of cell growth in animals. A long-time culture such as a few weeks or months may virtually exhibit a notable increase in cell proliferation. Nevertheless, our current in vitro proliferation showed the induction with statistical significance, supporting our hypothesis that lncRNA has tumor-promoting activity.

HuR was unexpectedly identified as a lncRNA-HGBCinteracting protein. There is a large body of evidence demonstrating that $\mathrm{HuR}$, a RNA binding protein, stabilizes mRNAs by binding to conserved AU-rich elements (AREs) within 3'UTRs and preventing gene degradation [46]. Nevertheless, HuR has been reported to be highly elevated in a number of cancers, such as brain tumor 
[47] and colon cancer [48]. Intriguingly, we also found that HuR can stabilize lncRNA-HGBC via 1759-1906 nt motif, which constitutes a positive feedback to rigorously stimulate lncRNA-HGBC expression and enhance tumor cell invasiveness. Indeed, we unveiled that HuR expression was upregulated and positively correlated with lncRNA-HGBC expression in GBC tissue. Therefore, our study has provided additional novel mechanistic insight into $\mathrm{HuR}$ in the regulation of lncRNA that drives GBC progression.

EMT is the key step mediating malignant transformation of a broad spectrum of cancers, including GBC [49]. We found that IncRNA-HGBC-induced metastasis was also associated with tumor cell EMT. EMT is genetically characterized by loss of epithelial cell biomarkers such as E-cadherin, but acquisition of mesenchymal proteins including $\mathrm{N}$-cadherin, vimentin, smooth alpha actin and Slug. In line with these genetically phenotypic alterations, GBC cells strongly expressed $\mathrm{N}$-cadherin and vimentin, and increased aggressive activity (cell migration and invasion). However, we currently can not exclude the possibility that these mesenchyme-associated proteins acquired by invasive tumor cells may be directly or indirectly regulated by miR-502-3p, HuR or SET. Thereof, it should be quite interesting to decipher potentially mechanistic link between EMT genes and lncRNA-HGBC downstream effectors.

\section{Conclusions}

In summary, we identified a novel lncRNA-HGBC, which is stabilized by $\mathrm{HuR}$, and acts as an endogenous sponge of miR-502-3p to promote GBC cell proliferation and metastasis. Inhibition of miR-502-3p upregulates SET which activates AKT signaling, resulting in cancer progression (Fig. 7j). The present study demonstrates that IncRNA-HGBC/miR-502-3p/SET/AKT axis plays a crucial role in GBC progression, pointing to lncRNA$\mathrm{HGBC}$ as a potential therapeutic target for GBC.

\section{Supplementary information}

Supplementary information accompanies this paper at https://doi.org/10. 1186/s12943-019-1097-9.

Additional file 1: Table S1. Sequence information used in this study. Table S2. Sequences of primers used for QRT-PCR in this study. Table S3. Primary antibodies used in this study. Table S4. Genes connected to IncRNA-HGBC. Table S5. The potential IncRNA-HGBC-interacting proteins identified by mass spectrometry between 30 and 40 KD. Table S6. Predicted InCRNA-HGBC-interacting miRNAs.

Additional file 2. Supplementary Methods.

Additional file 3: Figure S1. Identification of InCRNA-HGBC and its noncoding nature analysis. Figure S2. LnCRNA-HGBC promotes GBC cell proliferation and tumor growth. Figure S3. LnCRNA-HGBC promotes the invasive capacity of GBC cells. Figure S4. LnCRNA-HGBC did not influence HuR expression. Figure S5. Effects of miR-502-3p on IncRNA-HGBC expression. Figure S6. SET is a direct target of miR-502-3p in GBC cells.
Figure S7. miR-502-3p inhibits GBC cell proliferation and invasion. Figure S8. Knockdown of SET inhibits GBC cell proliferation and invasion. Figure S9. HuR is upregulated in GBC tissues.

\section{Abbreviations}

AGO2: Argonaute 2; ARE: AU-rich element; ceRNA: competing endogenous RNA; EMT: Epithelial-mesenchymal transition; FISH: Fluorescence in situ hybridization; FOXA1: Forkhead box A1; GAPDH: Glyceraldehyde-3-phosphate dehydrogenase; GBC: Gallbladder carcinoma; HuR: Hu antigen R; IHC: Immunohistochemistry; IncRNA-HGBC: long non-coding RNA Highly expressed in gallbladder carcinoma; MRE: MiRNA response element; MW: Molecular weight; PCNA: Proliferating cell nuclear antigen; qRTPCR: quantitative real-time PCR; RACE: Rapid amplification of the CDNA ends; RIP: RNA immunoprecipitation; SET: SET nuclear proto-oncogene

\section{Acknowledgements}

Not applicable.

\section{Authors' contributions}

YPH, XSW, YPJ, YY, YSL, HFL, SSX, XLS, L, YJZ and WH performed experiments; FTL, CC and QZ performed bioinformatical analysis; YPH, RS and YBL designed and analyzed experiment; YBL and HZC conceived and supervised the study and wrote the manuscript together with YPH and RS. All authors read and approved the final manuscript.

\section{Funding}

This study was supported by the National Natural Science Foundation of China (No. 31601021, 31620103910, 81874181, 91440203, 81772512

81672404), the Emerging Frontier Program of Hospital Development Center (No. SHDC12018107), the General Surgery Construction Program of Shanghai Municipal Health Commission (No. 2017ZZ02011), Xinhua Hospital Pl-startup funds (No. JZPI201716), the Project of Excellent Young Scholars from Shanghai Municipal Health and Family Planning Commission (No. 2018YQ10), the Talent Development Fund from Shanghai Municipal Human Resources and Social Security Bureau (No. 2018048), Shanghai Pujiang Program (No.

17PJ1406100) and Shanghai Key Laboratory of Biliary Tract Disease Research Foundation (No. 17DZ2260200).

\section{Availability of data and materials}

The datasets used and/or analyzed during the current study are available from the corresponding author on reasonable request.

\section{Ethics approval and consent to participate}

Written informed consent was obtained from all GBC patients. This study was approved by the ethics committee of Xinhua hospital. All the animal experiments were conducted strictly according to the Guide for the Care and Use of Laboratory Animals, and approved by the Animal Care and Use Committee of the Xinhua Hospital.

Consent for publication

All subjects have written informed consent.

\section{Competing interests}

The authors declare that they have no competing interests.

\section{Author details}

'Department of General Surgery, Xinhua Hospital, Affiliated to Shanghai Jiao Tong University School of Medicine, Building 25, Room 513, 1665 Kongjiang Road, Shanghai 200092, China. ${ }^{2}$ Shanghai Key Laboratory of Biliary Tract Disease Research, 1665 Kongjiang Road, Shanghai 200092, China. ${ }^{3}$ Shanghai Research Center of Biliary Tract Disease, 1665 Kongjiang Road, Shanghai 200092, China. ${ }^{4}$ Department of Pharmacology, Shanghai Jiao Tong University School of Medicine, W. Building 3, Room 407, 280 Chongqi Road, Shanghai 200025, China 
Received: 24 June 2019 Accepted: 4 November 2019

\section{10.0010}

\section{References}

1. Torre LA, Bray F, Siegel RL, Ferlay J, Lortet-Tieulent J, Jemal A. Global cancer statistics, 2012. CA Cancer J Clin. 2015;65:87-108.

2. Misra S, Chaturvedi A, Misra NC, Sharma ID. Carcinoma of the gallbladder. Lancet Oncol. 2003;4:167-76.

3. Batra Y, Pal S, Dutta U, Desai P, Garg PK, Makharia G, et al. Gallbladder cancer in India: a dismal picture. J Gastroenterol Hepatol. 2005;20:309-14.

4. Maibenco DC, Smith JL, Nava HR, Petrelli NJ, Douglass HO. Carcinoma of the gallbladder. Cancer Investig. 1998;16:33-9.

5. Tazuma S, Kajiyama G. Carcinogenesis of malignant lesions of the gall bladder. The impact of chronic inflammation and gallstones. Langenbeck's Arch Surg. 2001;386:224-9.

6. Lazcano-Ponce EC, Miquel JF, Munoz N, Herrero R, Ferrecio C, Wistuba II, et al. Epidemiology and molecular pathology of gallbladder cancer. CA Cancer J Clin. 2001:51:349-64

7. Wu XS, Shi LB, Li ML, Ding Q, Weng H, Wu WG, et al. Evaluation of two inflammation-based prognostic scores in patients with resectable gallbladder carcinoma. Ann Surg Oncol. 2014;21:449-57.

8. Butte JM, Matsuo K, Gonen M, D'Angelica MI, Waugh E, Allen PJ, et al. Gallbladder cancer: differences in presentation, surgical treatment, and survival in patients treated at centers in three countries. J Am Coll Surg. 2011;212:50-61.

9. Li M, Zhang Z, Li X, Ye J, Wu X, Tan Z, et al. Whole-exome and targeted gene sequencing of gallbladder carcinoma identifies recurrent mutations in the ErbB pathway. Nat Genet. 2014;46:872-6.

10. Maurya SK, Tewari M, Mishra RR, Shukla HS. Genetic aberrations in gallbladder cancer. Surg Oncol. 2012;21:37-43.

11. Wu H, Yang L, Chen LL. The diversity of long noncoding RNAs and their generation. Trends Genet. 2017;33:540-52.

12. Guttman M, Russell P, Ingolia NT, Weissman JS, Lander ES. Ribosome profiling provides evidence that large noncoding RNAs do not encode proteins. Cell. 2013;154:240-51.

13. Quinn JJ, Chang HY. Unique features of long non-coding RNA biogenesis and function. Nat Rev Genet. 2016;17:47-62.

14. Schmitt AM, Chang HY. Long noncoding RNAs in Cancer pathways. Cancer Cell. 2016;29:452-63.

15. Wapinski O, Chang HY. Long noncoding RNAs and human disease. Trends Cell Biol. 2011;21:354-61.

16. Flynn RA, Chang HY. Long noncoding RNAs in cell-fate programming and reprogramming. Cell Stem Cell. 2014;14:752-61.

17. Tay Y, Rinn J, Pandolfi PP. The multilayered complexity of ceRNA crosstalk and competition. Nature. 2014;505:344-52

18. Lu MH, Tang B, Zeng S, Hu CJ, Xie R, Wu YY, et al. Long noncoding RNA BC032469, a novel competing endogenous RNA, upregulates hTERT expression by sponging miR-1207-5p and promotes proliferation in gastric cancer. Oncogene. 2016;35:3524-34

19. Cao C, Zhang T, Zhang D, Xie L, Zou X, Lei L, et al. The long non-coding RNA, SNHG6-003, functions as a competing endogenous RNA to promote the progression of hepatocellular carcinoma. Oncogene. 2017;36:1112-22.

20. Qu L, Ding J, Chen C, Wu ZJ, Liu B, Gao Y, et al. Exosome-transmitted IncARSR promotes Sunitinib resistance in renal Cancer by acting as a competing endogenous RNA. Cancer Cell. 2016;29:653-68.

21. Lee GL, Dobi A, Srivastava S. Prostate cancer: diagnostic performance of the PCA3 urine test. Nat Rev Urol. 2011:8:123-4.

22. Prensner JR, Chinnaiyan AM. The emergence of IncRNAs in cancer biology. Cancer Discov. 2011;1:391-407.

23. Wu XS, Wang F, Li HF, Hu YP, Jiang L, Zhang F, et al. LncRNA-PAGBC acts as a microRNA sponge and promotes gallbladder tumorigenesis. EMBO Rep. 2017:18:1837-53.

24. Kertesz M, lovino N, Unnerstall U, Gaul U, Segal E. The role of site accessibility in microRNA target recognition. Nat Genet. 2007;39(10):1278-84

25. Wang L, Park HJ, Dasari S, Wang S, Kocher JP, Li W. CPAT: coding-potential assessment tool using an alignment-free logistic regression model. Nucleic Acids Res. 2013;41:e74

26. Hanahan D, Weinberg RA. Hallmarks of cancer: the next generation. Cell. 2011;144:646-74

27. Wang F, Yuan JH, Wang SB, Yang F, Yuan SX, Ye C, et al. Oncofetal long noncoding RNA PVT1 promotes proliferation and stem cell-like property of hepatocellular carcinoma cells by stabilizing NOP2. Hepatology. 2014;60: 1278-90.

28. Wu ZR, Yan L, Liu YT, Cao L, Guo YH, Zhang Y, et al. Inhibition of mTORC1 by IncRNA H19 via disrupting 4E-BP1/raptor interaction in pituitary tumours. Nat Commun. 2018;9(1):4624

29. Wang $L$, Zhao $Y$, Bao X, Zhu X, Kwok YK, Sun K, et al. LncRNA Dum interacts with Dnmts to regulate Dppa2 expression during myogenic differentiation and muscle regeneration. Cell Res. 2015;25(3):335-50.

30. Lebedeva S, Jens M, Theil K, Schwanhausser B, Selbach M, Landthaler M, et al. Transcriptome-wide analysis of regulatory interactions of the RNAbinding protein HuR. Mol Cell. 2011:43(3):340-52.

31. Qin W, Li X, Xie L, Li S, Liu J, Jia L, et al. A long non-coding RNA, APOA4-AS, regulates APOA4 expression depending on HuR in mice. Nucleic Acids Res. 2016;44(13):6423-33

32. Ale-Agha N, Galban S, Sobieroy C, Abdelmohsen K, Gorospe M, Sies H, et al. HuR regulates gap junctional intercellular communication by controlling betacatenin levels and adherens junction integrity. Hepatology. 2009:50(5):1567-76.

33. Shu YJ, Bao RF, Jiang L, Wang Z, Wang XA, Zhang F, et al. MicroRNA-29c-5p suppresses gallbladder carcinoma progression by directly targeting CPEB4 and inhibiting the MAPK pathway. Cell Death Differ. 2017;24(3):445-57.

34. Rupaimoole R, Calin GA, Lopez-Berestein G, Sood AK. miRNA deregulation in Cancer cells and the tumor microenvironment. Cancer Discov. 2016:6(3):235-46.

35. Jin H, Yu M, Lin Y, Hou B, Wu Z, Li Z, et al. MiR-502-3P suppresses cell proliferation, migration, and invasion in hepatocellular carcinoma by targeting SET. Onco Targets Ther. 2016;9:3281-9.

36. Hung $\mathrm{MH}$, Chen YL, Chu PY, Shih CT, Yu HC, Tai WT, et al. Upregulation of the oncoprotein SET determines poor clinical outcomes in hepatocellular carcinoma and shows therapeutic potential. Oncogene. 2016;35(37):4891-902.

37. Christensen DJ, Chen Y, Oddo J, Matta KM, Neil J, Davis ED, et al. SET oncoprotein overexpression in B-cell chronic lymphocytic leukemia and non-Hodgkin lymphoma: a predictor of aggressive disease and a new treatment target. Blood. 2011;118(15):4150-8.

38. Louro R, Smirnova AS, Verjovski-Almeida S. Long intronic noncoding RNA transcription: expression noise or expression choice? Genomics. 2009;93(4):291-8.

39. Salmena L, Poliseno L, Tay $Y$, Kats L, Pandolfi PP. A ceRNA hypothesis: the Rosetta stone of a hidden RNA language? Cell. 2011;146(3):353-8.

40. Wang YF, Yang L, Chen TX, Liu X, Guo Y, Zhu QJ, et al. A novel IncRNA MCM3AP-AS1 promotes the growth of hepatocellular carcinoma by targeting miR-194-5p/FOXA1 axis. Mol Cancer. 2019;18:28-43.

41. Sun M, Nie F, Wang Y, Zhang Z, Hou J, He D, et al. LncRNA HOXA11-AS promotes proliferation and invasion of gastric Cancer by scaffolding the chromatin modification factors PRC2, LSD1, and DNMT1. Cancer Res. 2016;76:6299-310.

42. Saddoughi SA, Gencer S, Peterson YK, Ward KE, Mukhopadhyay A, Oaks J, et al. Sphingosine analogue drug FTY720 targets I2PP2A/SET and mediates lung tumour suppression via activation of PP2A-RIPK1-dependent necroptosis. EMBO Mol Med. 2013;5:105-21.

43. Li J, Yang XF, Ren XH, Meng XJ, Huang HY, Zhao QH, et al. Stable SET knockdown in breast cell carcinoma inhibits cell migration and invasion. Biochem Biophys Res Commun. 2014;453:7-12.

44. Li M, Makkinje A, Damuni Z. The myeloid leukemia-associated protein SET is a potent inhibitor of protein phosphatase 2A. J Biol Chem. 1996;271:11059-62.

45. Kuo YC, Huang KY, Yang CH, Yang YS, Lee WY, Chiang CW. Regulation of phosphorylation of Thr-308 of Akt, cell proliferation, and survival by the B55alpha regulatory subunit targeting of the protein phosphatase $2 \mathrm{~A}$ holoenzyme to Akt. J Biol Chem. 2008:283:1882-92.

46. Brennan CM, Steitz JA. HuR and mRNA stability. Cell Mol Life Sci. 2001:58:266-77.

47. Nabors LB, Gillespie GY, Harkins L, King PH. HuR, a RNA stability factor, is expressed in malignant brain tumors and binds to adenine- and uridine-rich elements within the $3^{\prime}$ untranslated regions of cytokine and angiogenic factor mRNAs. Cancer Res. 2001:61:2154-61.

48. Dixon DA, Tolley ND, King PH, Nabors LB, Mclntyre TM, Zimmerman $G A$, et al. Altered expression of the mRNA stability factor HuR promotes cyclooxygenase-2 expression in colon cancer cells. J Clin Invest. 2001;108:1657-65.

49. Chang Y, Liu C, Yang J, Liu G, Feng F, Tang J, et al. MiR-20a triggers metastasis of gallbladder carcinoma. J Hepatol. 2013;59:518-27.

\section{Publisher's Note}

Springer Nature remains neutral with regard to jurisdictional claims in published maps and institutional affiliations. 